\title{
The Effect of Working Memory Capacity on Second Language Acquisition
}

\author{
Yulu Chai ${ }^{1, *}$ \\ ${ }^{1}$ Department of Experimental Psychology, University College London, London, United Kingdom, \\ *Corresponding author. Email: luluchai2000@gmail.com
}

\begin{abstract}
Second language acquisition (SLA) ability had been widely associated to cognitive abilities such as working memory (WM) capacity, where greater SLA ability is proposed to be positively correlated to WM capacity, implying there is a WM advantage in SLA. Hence, greater WM capacity may aid SLA. However, there were large discrepancies between the results of past studies on this topic, therefore, this study explores the relationship between SLA and WM capacity through evaluating past literature, where differences in methodology and data analysis are examined across studies. Results had found a small but robust effect size of WM capacity on SLA, especially in beginner and intermediate language learners, where the WM advantage in SLA weakens as language proficiency enhances. Implications of this paper could be extended to aid the development of new interventions supporting SLA, such as using training exercises to enhance WM capacity, especially for those with difficulties in SLA.
\end{abstract}

Keywords: working memory, second language acquisition, language acquisition, bilingualism, language development, language performance

\section{INTRODUCTION}

Second language learning and assessments have been part of the school curriculum and education systems across many countries, with many schools requiring their students to take at least one second language subject, which leads to an increasing number of people who are able to speak two or more languages [1]. Therefore, what makes second language acquisition (SLA) and being multilingual so highly valued? There are many known social benefits of SLA, in which being fluent in another language opens wider employment opportunities and allows for better communication with people from other cultural background. Furthermore, research also suggests that there are cognitive benefits of SLA, where it may enhance problem solving skills, attention control and act as a form of cognitive reserve in delaying the onset of dementia [2, 3, 4]. Due to the need to understand grammatical structures and remember vocabularies in order to speak any language, working memory (WM) capacity may be essential in SLA, where simultaneous processing of two or more languages may result in greater WM demand, thus WM capacity may be enhanced in the process of SLA, serving as a cognitive benefit $[5,6]$. However, research examining potential WM benefit in SLA showed discrepancies in their results, with some evidence supporting WM superiority in multilinguals over monolinguals and some suggesting no such benefit. Therefore, the first section of this review will focus on evaluating the extent of WM benefit in SLA, where SLA would be considered as a spectrum of second language ability, from early beginners to fluent bilinguals (regardless of whether they are early, learning two languages at birth, or late bilinguals, learning a second language after acquisition of the first).

\section{WM ADVANTAGE IN SLA}

Earlier research investigating the relationship between SLA and WM had provided evidence for second language superiority in performance in WM tasks. In a past study, a range of tasks with different WM demands were used to assess monolingual and bilingual participants [7]. The Simon task, where the participants were required to press one key for a certain stimulus and a different key for another stimulus, and the alpha span task, which presents two to eight words in a random order to the participants and required them to repeat back in alphabetical order, were used to assess WM capacity, with the Simon task considered as lighter in WM demand and the alpha span task as heavier. Results had found no significant difference in 
performance between the monolingual and bilingual participants in the lighter WM task, but a significant better performance of the bilinguals were found in the heavier WM task, highlighting potential bilingual advantage in WM over monolinguals in situations with greater WM demand. Furthermore, the alpha span task may also be a better measurement of WM with regard to language skills due to the high verbal working memory demand, hence reinforcing the link between SLA and WM capacity. However, the two languages that the participants spoke, either Tamil and English or Cantonese and English, were very different, therefore, the WM advantage concluded may not be generalisable to bilinguals speaking two languages from the same linguistic group, such as Spanish and Italian. Nonetheless, a similar study was conducted on multilinguals speaking similar languages, where their first language is Dutch, the second is German and had initiated their study in a third language, Norwegian [8]. A range of tasks with high or low WM demands were conducted and the multilinguals significantly outperformed the monolinguals in all tasks, regardless of their WM demands, which further supports the WM advantage in SLA. This study also demonstrated that such WM advantage can extend to a third language.

Similarly, a further study examined children aged five or seven with their native language as English, but varied in their second language, such as Arabic, Japanese and Spanish. Since, monolingual children typically demonstrate more vocabularies than the bilinguals in the language tested at a younger age, a non-verbal task with high WM demand, a computerised version of the traditional Corsi block task [8, 9], was chosen. The bilingual children did not show intergroup differences in task performance and performed better than the monolingual children overall, further reinforcing the proposed WM advantage of bilinguals and demonstrated that such advantage is evident irrelevant of the languages spoken. However, the sample size of each minor bilingual group was relatively small, therefore, the insignificant difference in task performance should be taken with caution and further investigation into the extent of WM benefit in different languages should be conducted with a greater sample size. Moreover, the studies discussed so far indicated a correlation between SLA and greater WM capacity and a causational link should not be concluded and thus the results should be interpreted with more caution.

On the other hand, some studies failed to provide evidence of WM advantage in SLA in finding no significant difference in WM task performance between monolinguals and bilinguals. French monolinguals, English monolinguals and French-English bilinguals were assessed by verbal and visual WM tasks and no significance difference in task performance were found between the bilinguals and monolinguals [10]. This lack of replication of WM advantage in bilinguals could be explained by the matched attention control abilities between the groups, suggesting that the observed WM advantage was not due to SLA, but better attention control often observed in bilinguals [4]. A correlation between performance on WM tasks and attention tasks was found, further supporting the relationship WM and attention, undermining the WM advantage in SLA. However, due to the attention control ability benefit of SLA and the correlation between attention control ability and WM, a WM benefit may be concluded, to some extent. Furthermore, the sample size of this study is relatively small, with 15 participants in each group, therefore, the reliability of the results may be undermined, as the insignificance may be due to the lack of power of the small sample size rather than true insignificance in task performance between the monolingual and bilingual children. Similarly, a verbal WM task was carried out in monolinguals and bilinguals, where no bilingual advantage was found [11]. Large effects sizes were found in the data analysis, reinforcing the lack of bilingual advantage in verbal WM. The lack of bilingual advantage may be explained by the assessment of solely simultaneous bilinguals, bilinguals that have learnt both languages simultaneously, who may be switching between different languages very naturally and would not require an as high WM demand [12]. Thus, previous studies concluding a bilingual advantage in WM may have examined sequential bilinguals, who have learnt a second language after being fluent in their first and hence may require greater WM demand and executive control to switch between the languages. Therefore, due to the large discrepancies between the results of different studies on this topic, more research may be required to determine the extent of SLA benefit in WM.

Nevertheless, more recent meta-analyses had suggested a significant SLA advantage in WM, whereby analysing a wide range of experiments allows for an examination of a bigger sample size over a range of conditions and hence may be able to produce more robust conclusions. A meta-analysis covering 27 studies concluded a small to medium effect size supporting the WM advantage in bilinguals [13], hence a correlation could be drawn between SLA and enhanced WM capacity. The meta-analysis also found that the WM advantage is the largest in children, which may be explained by the greater cognitive demand at earlier stages of language learning due to a lack of familiarity towards the language and hence more processing may be required. Moreover, a different meta-analysis assessing over a hundred studies drew similar conclusions, in which a small bilingual advantage in WM was found [14]. Due to the large discrepancies in results of previous studies, potential moderating factors such as the age of first exposure to the second language, proficiency of the second language and the WM 
demands of the tasks were considered during the analysis. Proficiency of the second language was found to contribute to the inconsistency regarding the extent of bilingual advantage in WM. However, the results of meta-analyses should be taken with caution due to potential accumulation of publication biases. Individual studies may be tempted to manipulate data into significant results to allow for greater chance of publishing, thus there may be an over-representation of such publication biases in the population of studies selected in the meta-analyses, which may result in overestimated effect sizes. Nonetheless, both meta-analyses discussed in this review carried out extra analysis of a fail-safe $\mathrm{N}$, both revealing that the population effect size may likely be safe from publication bias. Yet, both meta-analyses found a small to medium effect size, suggesting that the WM advantage observed in bilinguals may not be a great as claimed by individual studies.

\section{INDIVIDUAL DIFFERENCES IN WM ADVANTAGE}

Most research on the topic of SLA and WM focused on balanced bilinguals, who were exposed to two languages simultaneously, from an early age, and less research were based on sequential bilinguals, who learnt their second language after becoming native in their first, with different levels of proficiency in their second language. Therefore, since the large discrepancy in the results of previous studies examining WM and SLA was proposed to be due to the proficiency of the second language, the extent to which second language proficiency contributes to the individual differences over the WM advantage observed in past research should be further investigated. A previous study had found no WM advantage between bilinguals and monolinguals, but a significant difference was found between the monolinguals and the transitional bilinguals, where the individuals are native in one language and starting to learn another, and hence are at the early stages of becoming bilingual [15]. This may suggest an association between enhanced WM capacity and language development, where WM may be more involved in the initial learning and processing of languages. However, the definition of the bilingual subgroups, from transitional bilinguals to balanced bilinguals, were arbitrary, in which the proficiency of the second language was determined by scores on language proficiency tests with unjustified boundaries between beginner, intermediate and advanced speakers. Therefore, the results of this study should be interpreted with caution. Nonetheless, it provided a potential explanation to the differences observed between studies on this topic.

The relationship between language development and WM capacity was supported in a different study assessing English-Korean bilinguals with a range of second language proficiency [16]. By examining differences in WM capacity between Korean nearmonolinguals and English-Korean bilinguals, with intermediate and advance English proficiency determined by TOEFL scores, results found that the intermediate bilinguals out-performed the monolinguals and advanced bilinguals in both audio and visual digit span tasks with a large effect size, and no significant difference was found in the task performance between the monolinguals and the advanced bilinguals. This supported the idea that higher WM capacity is associated with learning and developmental stages of the second language. Structured interviews were also conducted with the participants to collect quantitative data for better understanding of the correlation found between second language development and enhanced WM capacity, where such correlation could be explained by the language practice differences between the intermediate and advance bilinguals. The advanced bilinguals reported less memorising and replaying than the intermediates as they may feel more natural and comfortable with their second language due to their higher fluency, which may imply a lesser extent of WM load in the advanced bilinguals and thus a lower WM capacity required. However, the conclusions drawn from the data may be limited to English-Korean bilinguals as the sentence and grammatical structure between English and Korean are very difference, which may lead to a greater WM demand to process and comprehend to the distinct languages, thus a significant difference in WM capacity may not be generalisable to bilinguals speaking two similar languages.

Furthermore, a longitudinal study on the WM capacity of first- and second-year undergraduates studying a second or third language revealed similar conclusion in which WM capacity is related to language development [17]. The participants' WM capacity was evaluated through a language-independent operation span task at the beginning of the school year and at the end of the second term. Significant improvements in performance on the WM task were found in both firstand second-year students, with a greater improvement observed in the first-year students, both in mean scores and effect size, which reinforces that WM abilities are more involved at the earlier stages of language acquisition. An additive improvement was also found in students learning their second and third languages simultaneously than those learning only one new language, which may be due to the greater WM demand required to process two new languages. This demonstrates that the WM advantage in SLA could be enhanced by the acquisition of more languages simultaneously, further supporting the correlation between language acquisition and enhanced WM capacity. However, the improvements observed in the WM task performance may be explained by confounds 
of practice. Nonetheless, due to the great time length between the first and second WM task assessments the bias of practise may be largely reduced and thus may not undermine the results obtained. However, there is one central limitation to all research on this topic as the correlation found between SLA and WM should not be interpreted as a causational link, therefore, the extent to which the enhanced WM capacity observed may be attributed to SLA cannot be determined and the direction of the correlation between the two, whether SLA leads to enhanced WM capacity or larger WM capacity results in better SLA, remains ambiguous. Therefore, more research is required to better understand the relationship between SLA and W and determine the direction of this observed correlation.

\section{EFFECT OF WM CAPACITY ENHANCEMENT}

A previous study had identified positive WM capacity effects on the learning of grammatical structures in SLA of beginners and intermediates, where limited WM capacity, often observed in adult second language learners, constraints the SLA of the learner [18]. Therefore, could training that enhances WM capacity be used as an intervention to aid SLA, especially for individuals with difficulty in SLA?

In a longitudinal experiment examining the effect of one-to-one WM training on SLA, first-year undergraduate Spanish students were recruited and placed into conditions with or without WM training [19]. Spanish grammar tests were conducted at the beginning and the end of the first academic term and score differences across the 2 tests were compared between groups, where no significant improvements in score was found between students in the condition with and without WM training. However, this insignificance may be attributed to the small sample size of the WM training group, with only 11 participants, the participation rate in the WM training sessions was very low which may further undermine the effect of WM training. The low participation rate may also indicate a lack intrinsic motivation, which may influence the effectiveness of the WM training, hence although an insignificance difference in test performance improvements was found between the two groups, a lack of effectiveness of the WM training in aiding SLA may not be concluded from the results and further research is required. This low participation may be resolved by introducing monetary compensations, thus future studies should consider using monetary or other forms of reward to motivate participants or enhance participation in order to collect more reliable data. Moreover, the experiment was conducted as part of an undergraduate thesis, which was not published in a formal academic journal, thus was not peer-reviewed.
Therefore, the results of this study should be taken with caution.

On the other hand, a similar study assessing the effect of WM training on performance in second language tests had found significant improvements in tests scores before and after six weeks of WM training using a variety of tasks, including introducing proverbs to the participants in their second language, which encouraging participants to associate existing knowledge, the story line of the proverb, with new knowledge, the vocabulary in the second language; and word association exercises, which also requires the association of new vocabularies to already known words [20]. All tasks used employs WM abilities to complete, thus function as WM training for the participants. The improvements observed in the participants' test scores indicated WM training may aid SLA, providing a direction for interventions to help people with SLA difficulties or those with a desire to improve their SLA. However, there are limitations to this experiment in which the sample size is extremely small, with only nine participants, which may undermine the power of the results obtained. Furthermore, the participants were selected for their poor performance in previous assessments of their second language and difficulty in SLA in general, thus the large effect size found in data analysis may be due to floor effects as they may have had a large room for improvements to start with. Therefore, the significant improvements found may not represent the effectiveness of the WM training. The results obtained also may not be generalisable to the wider population with no difficulty in SLA. Although the results of this study should be taken with caution and further experiments may be required to reinforce and consolidate its findings, the study provided a good foundation for future research in this area through demonstrating potential effect of WM training in aiding SLA and suggesting a range of possible WM training tasks that may be suitable for the domain of language acquisition.

Nevertheless, more research is required to examine potential effect of WM training in aiding and improving SLA, recruiting subjects with a wider range of existing second language proficiency and assessing a greater variety of potential WM training tasks. Due to the limited research on this topic, no reliable conclusion can be drawn on the effectiveness of WM training in aiding SLA. Nonetheless, existing literature may contribute to support the employment of WM trainings as an intervention for people who require SLA support.

\section{CONCLUSION}

This paper has discussed a range of literature focusing on the relationship between WM capacity and SLA. Despite discrepancies over the extent of WM 
advantage during SLA, evidence supporting the existence such WM advantage was more robust, therefore, it may be plausible to conclude a correlation between larger WM capacity and the process of SLA. Nonetheless, a causational link cannot be drawn between the two and hence the direction of the link between them remains unidentified. Further research examining the underlying factors contributing to the individual differences in WM capacity of bilinguals, which may explain for the discrepancies within the collection of studies assessing the effect of SLA on WM capacity, had identified second language proficiency as a contributor, where individuals with a lower second language proficiency may have a WM capacity superior to monolinguals and bilinguals with higher second language proficiency. This indicated a link between WM capacity and second language development, rather than general SLA. Such observation could be explained by the greater WM demand in earlier stages of SLA, where individuals are less familiar with the language and hence would require greater WM resource to process and comprehend to language; whereas in later stages of SLA, individuals may find the second language becoming more natural to them, and thus would require less mental rehearsing and process of the language and thus may employ less WM resources. Past studies had also found that limited WM capacity restricts SLA quality, providing a new direction into potential intervention for improving SLA through training that enhances WM capacity. However, this a relatively new area of research with very limited studies examining the effect of WM training on SLA, nonetheless, initial research supports the effectiveness of such training in aiding SLA. Further research is required in this field, where a greater range of conditions and larger samples sizes examined to produce reliable and robust conclusions on the effectiveness of WM training on SLA. Research in this area would have useful implications, in which it may help those with SLA difficulty, such as adult second language learners who may find SLA more difficult, to enhance their efficiency in language acquisition. Future studies should consider assessing the extent to which WM training improves SLA and examining the effectiveness of potential WM training that are the most suitable for enhancing SLA.

\section{AUTHORS' CONTRIBUTIONS}

This paper is independently completed by Yulu Chai.

\section{ACKOWLEDGMENTS}

I would like to express my sincere gratitude to Professor D. Szucs for his invaluable expertise in helping me to formulate the research topic, for without his guidance, this paper would not be possible. I would also like to Mina Zhou and all other staff for their guidance on my research direction and for their patience and support throughout my time writing this paper.

\section{REFERENCES}

[1] American Community Survey. (2015). Detailed languages spoken at home and ability to speak English for the population 5 years and over: 2009 2013.

[2] Bialystok, E. (2006). Effect of bilingualism and computer video game experience on the Simon task. Canadian Journal of Experimental Psychology, 60(1), 68-79.

[3] Bialystok, E., \& Craik, F. (2010). Cognitive and Linguistic Processing in the Bilingual Mind. Current Direction in Psychological Science, 19(1), 19-23.

[4] Craik, F., Bialystok, E., \& Freedman, M. (2010). Delaying the onset of Alzheimer disease: Bilingualism as a form of cognitive reserve. Neurology, 75(19), 1726-1729.

[5] Mickan, A., McQueen, J., \& Lemhöfer, K. (2019). Bridging the gap between second language acquisition research and memory science: The case of foreign language attrition. Frontiers in Human Neuroscience, 13, 397-397.

[6] de Bot, K. (2012). Rethinking multilingual processing : From a static to a dynamic approach. In S. Flynn, J. Rothman, \& J. Cabrelli Amara, hird language acquisition in adulthood (pp. 79 - 93). John Benjamins Publishers.

[7] Bialystok, E., Craik, F., Klein, R., \& Viswanathan, M. (2004). Bilingualism, aging and cognitive control: Evidence from the Simon task. Psychology and Aging, 19(2), 290-303.

[8] Bialystok, E., Luk, G., Peets, K., \& Yang, S. (2010). Receptive vocabulary differences in monolingual and bilingual children. Bilingualism, 13(4), 525531.

[9] Van Den Noort, M., Bosch, P., \& Hugdah, K. (2006). Foreign language proficiency and working memory capacity. European Psychologist, 11(4), 289-296.

[10] Berch, D., Krikorian, R., \& Huha, E. (1992). The Corsi block-tapping task: Methodological and theoretical considerations. Brain and Cognition, 38(3), 317-338.

[11] Engel de Abreu, P. (2011). Working memory in multilingual children: Is there a bilingual effect? Memory, 19(5), 529-537. 
[12] Namazi, M., \& Thordardottir, E. (2010). A working memory, not bilingual advantage, in controlled attention. International Journal of Bilingual Education and Bilingualism, 13(5), 597616.

[13] Grundy, J., \& Timmer, K. (2017). Bilingualism and working memory capacity: A comprehensive meta-analysis. Second Language Research, 33(3), 325-340.

[14] Monnier, C., Boiché, J., Armandon, P., Baudoin, S., \& Bellocchi, S. (2021). Is bilingualism associated with better working memory capacity? A meta-analysis. International Journal of Bilingual Education and Bilingualism, 1-27.

[15] Kudo, M., \& Lee, S. (2014). Are there advantages for additive bilinguals inworking memory tasks? Learning and Individual Differences, 35, 96-102.

[16] Yang, E. (2017). Bilinguals' working memory (WM) advantage and their dual language practices. Brain Sciences, 7(7), 1-21.

[17] Huang, T., Loerts, H., \& Steinkrauss, R. (2020). The impact of second- and third-language learning on language aptitude and working memory. International Journal of Bilingual Education and Bilingualism, 1-17.

[18] Serafini, E., \& Sanz, C. (2016). Evidence for the decreasing impact of cognitive ability on second language development as proficieny increases. Studies in Second Language Acquisition, 38(4), 607-646.

[19] Godes, J. (2019). Second Language Acquisition and Memory Training Transfer: Could working memory training facilitate the learning of a second language? UVM Honors College Senior Theses.

[20] Santacruz, D., Dávila, H., \& Castillo, E. (2020). Games for working memory training in foreign language learning. MEXTESOL Journal, 44, no.4. 